Reprod. Nutr. Dévelop., 1984, 24 (5A), 655-670.

\title{
Déafférentation vagale totale et évacuation gastrique chez le porc
}

\author{
J.-P. LAPLACE, J.-C. CUBER \\ avec la collaboration technique de Sylvette GOUGIS, Chantal KABORÉ, \\ Brigitte MOUROT et A. VAN HECKE
}

Laboratoire de Physiologie de la Nutrition, I.N.R.A., 78350 Jouy-en-Josas, France.

Summary. Total vagal deafferentation and gastric emptying in the pig.

50-kg pigs fed a purified wheat starch and casein diet were fitted with a gastric cannula. Gastric emptying was studied by checking the residual gastric contents at 30 min and 1, 2, 4 and $7 \mathrm{hrs}$ after feeding. Five of the 25 pigs were used as controls; 5 were submitted to total vagal deafferentation including removal of the left nodose ganglion, right truncal vagotomy at the diaphragm and resection of the interconnecting branches between the two vagi on their intrathoracic course ; 5 were submitted to total contralateral vagal deafferentation ; 5 only underwent left truncal vagotomy and resection of the connectives : 5 were submitted to the contralateral operation. Total vagal deafferentation, suppressing bilateral afferences originating below the diaphragm, resulted in much higher residual gastric contents at all times studied after the meal. Significant gastric retention was also observed after unilateral truncal vagotomy as compared to the controls. Nevertheless, this retention was significantly lower than after total deafferentation. The reduced emptying rate after total vagal deafferentation was a result of the loss of all afferences, while the simultaneous reduction of efferences was of little importance. The mechanisms responsible for gastric retention have been discussed in relation with the sustained antral hyperactivity previously found in deafferented pigs and with the various controls of gastric emptying. Several, factors may be involved, including both motor and secretory changes. The prolonged postprandial activity of the gastric antrum seemed to be a consequence of gastric retention.

\section{Introduction.}

La sensibilité viscérale digestive ne peut être sous estimée compte tenu du nombre de régulations physiologiques et comportementales dans lesquelles on reconnaît aujourd'hui sa mise en jeu (Mei, 1981). Si l'on ne considère que le seul nerf vague, les données morphologiques fournies par microscopie optique ou électronique montrent que les fibres sensitives afférentes en constituent la plus grande partie, soit environ $80 \%$ dans le cas précis du chat (Mei, Condamin et Boyer, 1980). Par ailleurs, les données électrophysiologiques permettent d'inven- 
torier au niveau de la paroi digestive divers types de récepteurs (Leek, 1977) en rapport avec des fibres afférentes vagales: mécano-récepteurs (Iggo, 1955; Mei, 1970b ; Andrews, Grundy et Scratcherd, 1980), chémorécepteurs sensibles à I'alcalinité, à l'acidité, au glucose et éventuellement à divers glucides (lggo, 1957 ; Mei, 1978 ; El Ouazzani et Mei, 1981), ou encore à un ou plusieurs acides aminés (Jeanningros et Mei, 1980), thermorécepteurs (El Ouazzani et Mei, 1979). Tous ces récepteurs vagaux, présents en grand nombre au niveau gastro duodénal, sont à l'origine de régulations réflexes qui assurent une coordination des activités respectives de l'estomac et du duodénum, et concourent avec divers peptides et hormones gastro intestinaux à une régulation neurohumorale de la motricité et de l'évacuation de l'estomac (Cooke, 1975 ; Rozé, 1980a et b).

Une démonstration du rôle du nerf vague dans le contrôle de l'évacuation de l'estomac est apportée par la disparition plus ou moins complète, après vagotomie tronculaire bilatérale, des effets inhibiteurs de perfusions duodénales d'acide chlorhydrique, de glucose ou d'huile d'olive sur la motricité gastrique du porc (Roze et al., 1977). Cependant, la vagotomie tronculaire interrompt à la fois la conduction des influx afférents et efférents. Bien que les fibres afférentes et efférentes soient mélangées dans le nerf vague comme dans la plupart des nerfs viscéraux, il existe dans certaines espèces telles que le chat (Mei, 1970a), le porc (Darcy et al., 1979) et même le mouton (Darcy et al., 1979 ; Falempin et Rousseau, 1979), une séparation anatomique quasi complète des deux types de fibres au niveau du ganglion plexiforme. Cette disposition particulière permet, tout en préservant partiellement les efférences, une suppression des afférences vagales, subtotale (Darcy et al., 1979) ou totale (Laplace, 1980a). Paradoxalement, il a été observé antérieurement que la déafférentation vagale subtotale, supposée interrompre les réflexes inhibiteurs qui participent au contrôle de l'évacuation, ralentit considérablement celle-ci chez le porc (Laplace, Cuber et Villiers, 1979).

Compte tenu de ces éléments, le présent travail a pour objet de décrire les effets de la déafférentation totale sur l'évacuation gastrique chez le porc.

\section{Matériel et méthodes.}

Au total 25 porcs, mâles castrés, de race Large White, ont été utilisés en deux séries expérimentales consécutives. L'une a comporté trois groupes de cinq animaux désignés comme suit : témoins, déafférentés dits de type $G$, déafférentés dit de type D. L'autre a mis en œuvre deux groupes de cinq porcs dits tronculaires $D$ et tronculaires $G$. Le poids vif moyen des 25 porcs lors de l'opération était de $49,1 \pm 0,5 \mathrm{~kg}$. Leur gain moyen quotidien de poids vif au cours de la période expérimentale (21 à 24 jours) a été de $483 \pm 32 \mathrm{~g}$.

1. Préparation chirurgicale des animaux. - Sous anesthésie générale à I'halothane tous les porcs subissent, selon la technique antérieurement décrite (Cuber, Laplace et Villiers, 1980), une fistulation de l'estomac qui est la seule intervention pratiquée chez les porcs témoins.

Au cours de la même anesthésie, dix porcs subissent en plus la déafférentation vagale dite totale (Laplace, 1980a). Cette intervention comporte pour cinq 
d'entre eux (type G) les trois étapes suivantes : ablation du ganglion plexiforme gauche par voie rétroparotidienne, vagotomie tronculaire droite au niveau du diaphragme et ablation, à la faveur d'une thoracotomie, des rameaux reliant entre eux les deux vagues thoraciques. Pour les cinq autres porcs (type D), la déafférentation est réalisée " en miroir " avec ablation du ganglion plexiforme droit et vagotomie tronculaire gauche. Le résultat de ces opérations, qui suppriment toute afférence mais préservent selon le cas une voie vagale efférente gauche (G) ou droite (D) est schématisé dans la figure 1.

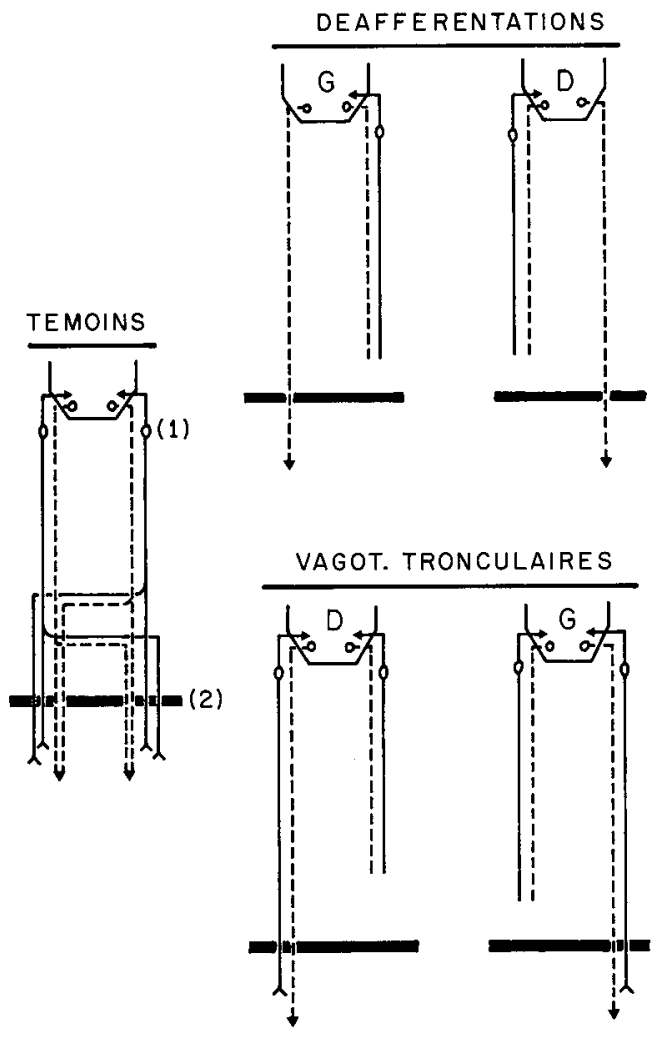

FIG. 1. - Représentation schématique de la disposition des voies vagales afférentes (trait continu) et efférentes (trait pointillé) chez l'animal intact, après les déafférentations totales dites gauche ou droite, et après les vagotomies tronculaires homologues, droite ou gauche; $(1)=$ ganglion plexiforme, $(2)=$ diaphragme.

Dans la seconde série expérimentale les dix porcs ont été soumis, également au cours de la même anesthésie que pour la fistulation gastrique, à une vagotomie tronculaire unilatérale au niveau du diaphragme avec ablation des rameaux anastomotiques unissant les deux troncs vagaux. L'intervention (fig. 1) est pratiquée soit à droite (tronculaires $\mathrm{D}$ ), les résultats fournis par ces cinq porcs pouvant 
être comparés à ceux des déafférentés $G$, soit à gauche (cinq tronculaires $\mathrm{G}$ ) pour comparaison aux déafférentés $D$.

Après l'intervention chirurgicale, les porcs sont installés individuellement en cage à métabolisme et réalimentés progressivement avec l'aliment d'épreuve. L'expérimentation proprement dite débute au plus tôt 8 jours après l'opération.

2. Aliment et mode de distribution. - L'aliment expérimental est un régime semi purifié, formulé pour un taux de matières azotées totales de $14 \%$ et qui apporte $4463 \mathrm{kcal}$ par $\mathrm{kg}$ de matière sèche. Ses constituants sont les suivants (proportion en \%) : amidon de blé purifié $(72,3)$, caséine chlorhydrique $(16,1)$, cellulose de bois purifiée $(6,0)$, huile d'arachide $(2,0)$, minéraux $(2,5)$, vitamines $(1,0)$, anti oxydant $(0,1)$. Cet aliment est, pendant toute la période expérimentale, systématiquement distribué en 2 repas quotidiens à $9 \mathrm{~h}$ et $16 \mathrm{~h}$. Chacun d'eux apporte $1 \mathrm{~kg}$ de farine fraîche diluée par 1,5 I d'eau. L'eau de boisson est apportée en totalité par l'eau de dilution de l'aliment en l'absence de tout abreuvement complémentaire.

3. Collecte des digesta et méthodes analytiques. - L'évaluation de l'évacuation gastrique est fondée sur la mesure des quantités résiduelles dans l'estomac à divers temps postprandiaux, par la collecte totale des contenus gastriques (Cuber, Laplace et Villiers, 1980). Celle-ci est pratiquée dans tous les cas à la suite du repas du matin. La vacuité gastrique est contrôlée préalablement au repas d'épreuve par abreuvement fictif (canule gastrique ouverte). Une seule collecte des contenus gastriques résiduels est effectuée chaque jour, $30 \mathrm{~min}, 1 \mathrm{~h}, 2 \mathrm{~h}, 4 \mathrm{~h}$ ou $7 \mathrm{~h}$ après l'ingestion du repas. Aussitôt après la collecte, les porcs reçoivent un repas de remplacement équivalant à la quantité recueillie. Lorsque la collecte a lieu $7 \mathrm{~h}$ après le repas d'épreuve, le repas de remplacement est fourni en complément du second repas quotidien. Chaque animal est utilisé au total dix fois à raison de deux mesures pour chacun des cinq instants d'observation de façon à constituer deux « cinétiques " par porc.

Dans tous les cas, les contenus gastriques résiduels sont pesés, homogénéisés et échantillonnés en vue de la détermination de la teneur en matière sèche (24 h à l'étuve à $104^{\circ} \mathrm{C}$ ). Dans la première série expérimentale, on a également déterminé, après lyophilisation, les teneurs en azote total (méthode Kjeldahl) et en amidon d'autre part. L'amidon est défini comme l'ensemble du glucose et de tous ses polymères alpha, et dosé selon Cuber et Laplace (1979). Ces éléments permettent le calcul des quantités totales de matière sèche, et éventuellement d'amidon et d'azote, résiduelles dans l'estomac aux divers temps de collecte. Celles-ci sont exprimées en pourcentage des quantités ingérées, les mêmes déterminations étant effectuées sur les échantillons correspondants de l'aliment.

A titre complémentaire, chez les porcs de la première série expérimentale, les quantités résiduelles de matière fraîche et de matière sèche ont fait l'objet d'une mesure à l'occasion du contrôle de la vacuité préalable au repas d'épreuve du matin. Ces mesures fournissent donc une indication sur les contenus gastriques résiduels $17 \mathrm{~h}$ après le repas distribué le jour précédent à $16 \mathrm{~h}$. 


\section{Résultats.}

1. Effets de la déafférentation totale. - Les quantités résiduelles recueillies en moyenne (fig. 2) sont analogues, pour les quatre variables contrôlées et à tous les instants de mesure, chez les porcs déafférentés de type $G$ et de type $D$. Les écarts les plus importants entre valeurs moyennes restent toujours non significatifs (test $t$ de Student). Ces quantités résiduelles recueillies chez les porcs déafférentés sont significativement plus importantes que chez les porcs témoins $(P<$ 0,001 dans la plupart des cas) pour la matière sèche, l'amidon et l'azote résiduels. Par contre, les trois groupes de porcs présentent des quantités résiduelles de matière fraîche analogues à $30 \mathrm{~min}, 1 \mathrm{~h}$ et $2 \mathrm{~h}$; elles sont ensuite significativement plus importantes chez les porcs déafférentés $(P<0,005$ à $7 \mathrm{~h})$.

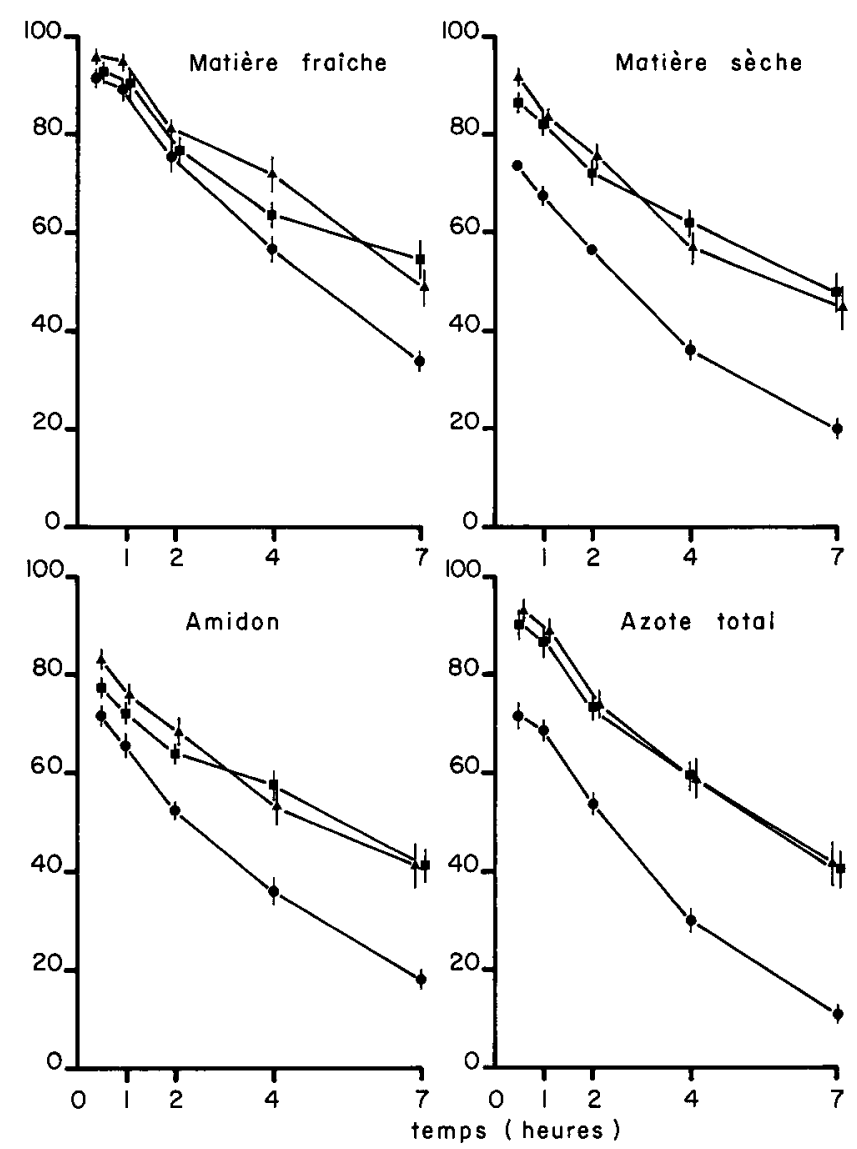

FIG. 2. - Quantités movennes résiduelles dans l'estomac, en \% des quantités ingérées correspondantes, à divers temps postprandiaux chez des porcs témoins (O) et chez des porcs soumis à déafférentation vagale totale de type $G(\mathbb{G})$ et de type $D(\mathbf{A})$. L'écart type de la moyenne est représenté lorsque sa valeur le permet. 
La variabilité des quantités résiduelles, exprimée par le coefficient de variation (fig. 3), reste, pour toutes les variables contrôlées et même chez les porcs déafférentés, égale ou inférieure à celle qui est habituellement observée pour ce type de mesures (Cuber, Laplace et Villiers, 1980). II n'apparaît pas de différences systématiques entre les 2 types de déafférentés, ni entre ceux-ci et les témoins.

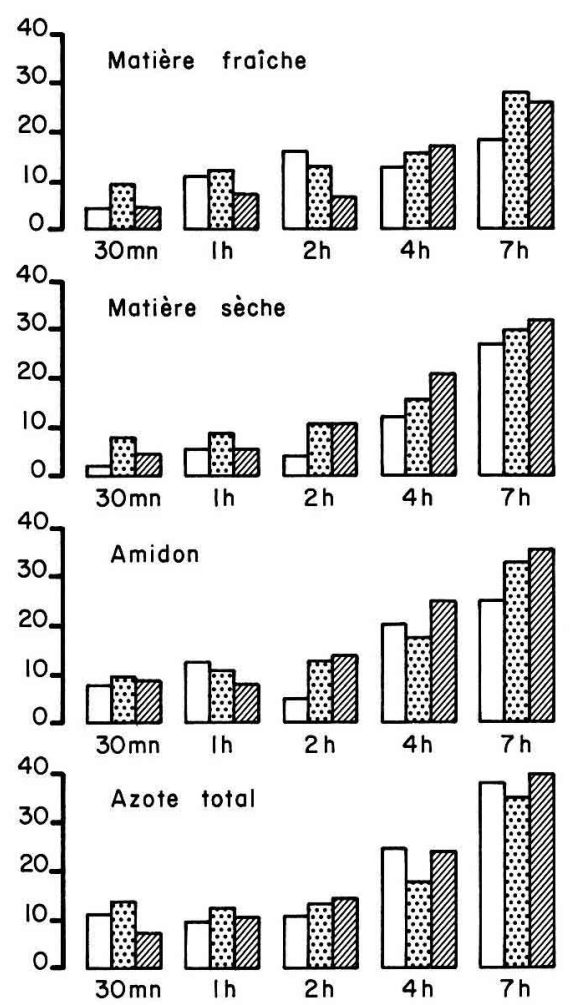

FIG. 3. - Variabilité des quantités résiduelles dans /'estomac (coefficient de variation en \%) aux divers instants de mesure chez des porcs témoins (blanc) et chez des porcs soumis à déafférentation vagale totale de type $G$ (pointillé) et de type $D$ (hachures).

Enfin, les contrôles effectués $17 \mathrm{~h}$ après le repas du soir montrent que l'estomac des porcs déafférentés contient encore une quantité de matière fraîche équivalant à plus de $30 \%$ des quantités ingérées, et une quantité de matière sèche égale au quart ( $G: 26,6 \pm 2,5 ; D: 24,2+2,2 \%)$ de la matière sèche ingérée, alors que l'estomac des porcs témoins ne renferme plus que quelques sécrétions correspondant à une quantité négligeable de matière sèche.

2. Effets de la vagotomie tronculaire. - Les quantités résiduelles moyennes de matière sèche recueillie aux divers instants de mesure sont analogues pour les porcs soumis à vagotomie tronculaire de type $D$ et de type $G$. Ces quantités sont significativement plus importantes que chez les porcs témoins : $p<0,001$ pour les 2 groupes à tous les temps de mesure sauf pour la vagotomie tronculaire 
droite $(\mathrm{P}<0,05$ à $7 \mathrm{~h})$. Mais elles restent inférieures à celles recueillies chez les porcs déafférentés (fig. 4). La signification de cet écart, pour la comparaison déafférentation gauche - tronculaire droite, est enregistrée au seuil $P<0,10$ à $1 \mathrm{~h}$ et $2 \mathrm{~h}$, et au seuil $\mathrm{p}<0,01$ à 4 et $7 \mathrm{~h}$; de même pour la comparaison déafférentation droite - tronculaire gauche les seuils de signification sont les suivants : 0,01 à $30 \mathrm{~min}, 0,10$ à $1 \mathrm{~h}, 0,01$ à $2 \mathrm{~h}$ et 0,05 à 4 et $7 \mathrm{~h}$. Par conséquent, tout en restant supérieure à ce qu'elle est chez les témoins, la rétention gastrique de la matière sèche après vagotomie tronculaire unilatérale est significativement moins importante que lors de déafférentation vagale.
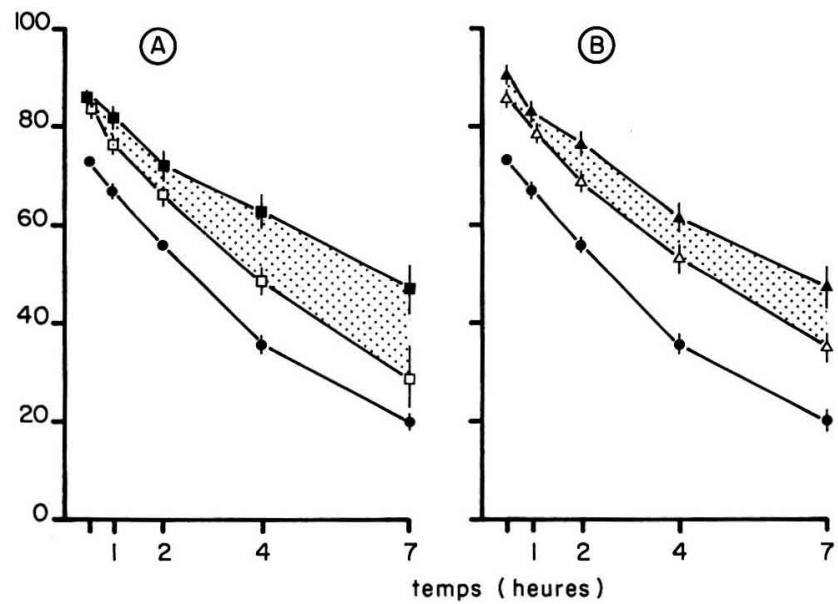

FIG. 4. - Quantités moyennes résiduelles de matière sèche dans l'estomac, en \% de la quantité ingérée, à divers temps postprandiaux chez des porcs témoins 101 , et chez les operrés: en $A$ : déafférentation $G(\square)$ et vagotomie tronculaire $D(\square)$; en $B$ : déafférentation $D(\Delta)$ et vagotomie tronculaire $G(\Delta)$.

La surface grisée estime la part de l'effet dû à l'ablation du seul ganglion plexiforme gauche (en $A$ ) ou droit (en $B$ ).

La variabilité des quantités résiduelles de matière sèche, exprimée par le coefficient de variation (fig. 5), est systématiquement moindre chez les porcs soumis à
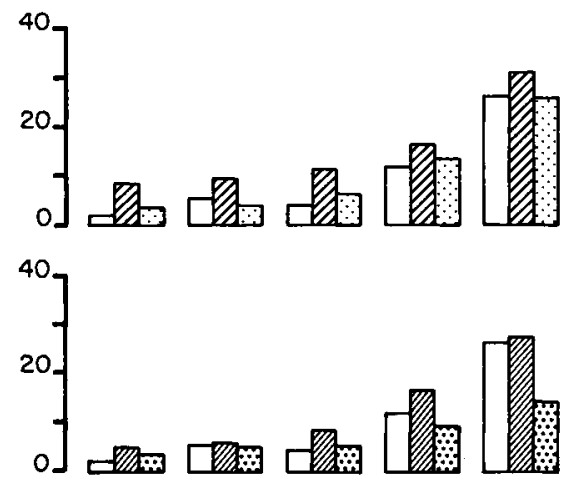

FIG. 5. - Variabilité des quantités de matière sèche résiduelle dans /'estomac (coefficient de variation en \%) aux divers instants de mesure chez les porcs témoins (blanc) et chez les opérés:

en haut: déafférentation $G$ (hachures) et vagotomie tronculaire $D$ (pointillé) ; en bas : déafférentation $D$ (hachures) et vagotomie tronculaire $\mathrm{G}$ (points). 
vagotomie tronculaire que chez les déafférentés homologues. Elle est même parfois analogue ou inférieure à celle enregistrée chez les porcs témoins. Aux temps 4 et $7 \mathrm{~h}$, elle est nettement plus grande après vagotomie tronculaire droite $(13,7$ et $26,0 \%)$ qu'après vagotomie tronculaire gauche $(9,8$ et $14,3 \%)$.

3. Evolution de la teneur en matière sèche des contenus gastriques. L'évolution, au cours des 7 heures postprandiales, de la teneur moyenne en matière sèche des contenus gastriques résiduels est très modifiée par les déafférentations ou par les vagotomies tronculaires (fig. 6). Cette teneur décroît au cours du temps de 28,2 à $21,0 \%$ chez les porcs témoins. Chez les porcs soumis à I'un ou l'autre des quatre types d'intervention sur les nerfs vagues, elle est très significativement plus élevée $(P<0,05$ au moins et $P<0,001$ dans la plupart des cas) et ne présente pas de décroissance systématique.

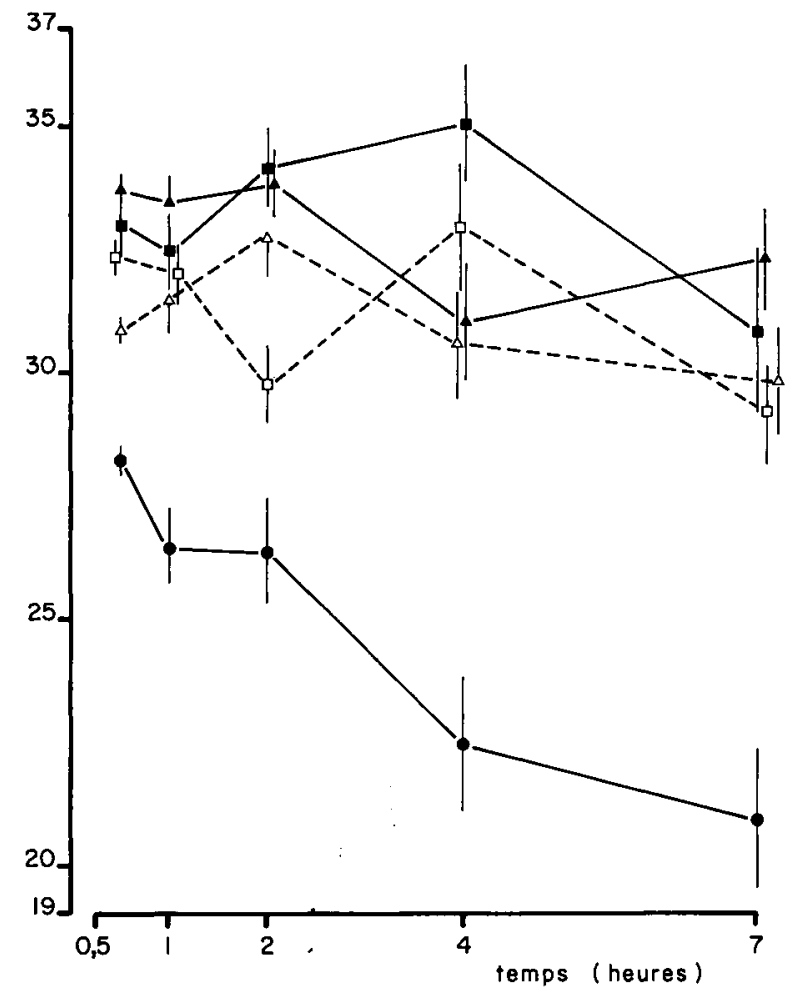

FIG. 6. - Teneur moyenne en matière sèche $(\%)$ des contenus gastriques résiduels aux divers instants de mesure, avec écart-type de la moyenne, selon le type d'intervention. Les symboles utilisés sont les mêmes que dans la figure 4 .

On peut encore noter que les teneurs enregistrées après vagotomie tronculaire droite sont toujours inférieures à celles mesurées chez les porcs soumis à la déafférentation gauche (comportant la même vagotomie tronculaire droite) et qu'il en va de même pour les interventions contralatérales. Mais ces différences ne 
sont significatives qu'au temps $2 \mathrm{~h}$ dans le premier cas, et au temps $30 \mathrm{~min}$ et $1 \mathrm{~h}$ dans le second.

\section{Discussion.}

\section{Importance respective des afférences et des efférences.}

Les résultats qui précèdent démontrent sans ambiguïté que la suppression chirurgicale de la totalité des afférences vagales d'origine post-diaphragmatique réduit considérablement les quantités évacuées par l'estomac au cours des $7 \mathrm{~h}$ consécutives au repas : la quantité résiduelle de matière sèche au temps $7 \mathrm{~h}$ chez les déafférentés représente en effet $47 \%$ de l'ingéré contre $20 \%$ seulement chez les témoins. Cet effet de la déafférentation totale confirme donc les observations préliminaires réalisées après déafférentation subtotale (Laplace, Cuber et Villiers, 1979). Ainsi, trois remarques d'intérêt méthodologique peuvent être formulées : (i) le contingent sensitif éventuellement préservé dans la déafférentation subtotale, à savoir celui qui atteindrait le vague cervical droit à partir du vague abdominal gauche $i$. e. ventral (ou leurs contralatéraux) par les anastomoses intrathoraciques entre les deux troncs, n'aurait qu'une importance fonctionnelle réduite dans le contrôle nerveux de l'évacuation gastrique ; (ii) la réduction supplémentaire du volume d'efférences, qui peut aussi résulter de la déafférentation totale en raison de la suppression de ces mêmes anastomoses, ne semble pas de nature à affecter sensiblement l'effecteur étudié ; (iii) l'analogie des effets enregistrés ici pour les deux types $G$ et $D$ de déafférentation totale suggère, en première analyse, une relative équivalence fonctionnelle des voies vagales, au moins à l'égard des fonctions motrices du territoire gastro duodénal impliqué dans le processus d'évacuation gastrique.

Ceci étant, la déafférentation totale est assurée en 3 temps opératoires dont I'un seulement (ablation du ganglion plexiforme) concerne exclusivement le versant afférent, tandis que les 2 autres (vagotomie tronculaire et section des anastomoses) affectent à la fois les versants afférent et efférent. L'intégralité de la rétention gastrique consécutive à la déafférentation totale ne peut donc être imputée avec certitude à la privation d'afférences. Par contre, la différence entre les résultats obtenus après déafférentation et ceux enregistrés après simple vagotomie tronculaire et section des rameaux anastomotiques, fournit une estimation des conséquences de l'ablation du seul ganglion plexiforme (plage hachurée de la figure 4). Si l'on note 100 l'effet de rétention induit au temps $7 \mathrm{~h}$ par la déafférentation de type $G$ par rapport aux valeurs témoins, on constate que $31,2 \%$ de cette rétention seraient imputables à la vagotomie tronculaire droite, tandis que $68,8 \%$ de l'effet de la déafférentation de type $G$ résulteraient de l'ablation du ganglion plexiforme gauche. Ces valeurs suggèrent aussi que l'importance fonctionnelle du ganglion plexiforme droit, privé d'afférences du fait de la vagotomie tronculaire droite et de la section des rameaux anastomotiques, est plus modeste dans la mesure où elle ne serait responsable que d'une fraction non précisée des $31,2 \%$ de la rétention consécutive à la vagotomie tronculaire droite. Si l'on procède aux mêmes évaluations dans le cas des interventions contralatérales, on 
observe effectivement que la rétention gastrique après déafférentation de type $D$ serait liée pour $57,1 \%$ à la vagotomie tronculaire gauche avec section des anastomoses et pour $42,9 \%$ seulement à l'ablation du ganglion plexiforme droit.

$\mathrm{Au}$ total, on peut donc retenir avec certitude que la privation d'afférences, liée à l'ablation d'un seul ganglion plexiforme, conduit à une rétention gastrique significative. On peut aussi retenir, même si les estimations qui précèdent n'ont qu'une valeur indicative (porcs différents dans chaque groupe, estimations au temps $7 \mathrm{~h}$ ), que le ganglion plexiforme gauche a très probablement une importance fonctionnelle plus grande que celle de son homologue contralatéral pour ce qui concerne l'effecteur gastro duodénal et le contrôle de l'évacuation gastrique. Enfin si l'on tient compte, dans l'effet très important de la déafférentation totale, telle qu'elle a été pratiquée, du fait que l'ablation d'un seul ganglion plexiforme répond d'une bonne part de la rétention et qu'une partie de l'effet complémentaire de la vagotomie tronculaire contralatérale est liée à la déconnection de l'autre ganglion plexiforme, on peut estimer par le calcul qu'environ $80 \%$ de l'effet des déafférentations seraient le fait de la privation d'afférences alors que $20 \%$ seulement résulteraient de l'inévitable réduction concomitante des efférences.

\section{Conséquences de la déafférentation.}

Au-delà de cette analyse, directement liée à la méthodologie, des responsabilités respectives des afférences et des efférences, il convient d'examiner également la nature des effets résultant de la déafférentation. Mais l'évaluation de l'influence exercée sur l'évacuation du repas d'épreuve ingéré est rendue délicate par l'interférence entre les apports de volumes d'origine sécrétoire et l'évacuation différentielle des solides et des liquides.

2.1. Effets sur la sécrétion gastrique. - Le fait que, durant les 2 premières heures postprandiales, les quantités résiduelles de matière sèche sont plus importantes chez les déafférentés que chez les témoins, alors que celles de matière fraîche sont analogues, est remarquable. Pour une teneur en matière sèche de l'aliment ingéré de $35 \pm 0,2 \%$, celle des contenus gastriques résiduels après $30 \mathrm{~min}$ est de $33,35 \%$ en moyenne chez les déafférentés contre $28,25 \%$ chez les témoins. II y a donc, par rapport à l'ingéré, une importante dilution de la matière sèche chez les témoins (teneur réduite de 7 points) en l'espace des 30 premières minutes postprandiales; cette dilution est beaucoup plus faible (teneur abaissée de 2 points) chez les déafférentés. Si l'on admet une même dilution par la sécrétion salivaire, il est vraisemblable que la déafférentation réduit fortement la sécrétion gastrique postprandiale, effet qu'il conviendra d'étudier directement. Ce phénomène apparemment limité à la période postprandiale immédiate ne peut cependant pas rendre compte de l'écart des quantités résiduelles selon les groupes, tant en matière fraîche qu'en matière sèche, au-delà de 2 heures.

2.2. Déafférentation et rétention gastrique. - La lenteur d'évacuation de l'estomac, observée ici pour un régime semi-purifié qui forme une bouillie fluide et homogène de fines particules, et chez des porcs dont la vacuité gastrique effective est assurée avant chaque épreuve, n'entraîne pas de désordres cliniques. Mais on conçoit qu'une rétention de cette importance puisse conduire à des troubles graves (distension abdominale, anorexie), voire à la mort dans $30 \%$ des cas 
comme nous l'avons observé (Laplace, 1980a) chez des porcs soumis à déafférentation totale, mais recevant un aliment grossier (céréales broyées, son...). Dans tous les cas, déafférentation subtotale ou totale et régime standard de texture grossière ou régime purifié de texture très fine, une hyperactivité antrale postprandiale prolongée (Darcy et al., 1979 ; Laplace, 1980a) coexiste avec une évacuation gastrique plus lente. II n'est donc pas possible d'interpréter ces phénomènes par la simple suppression d'un arc réflexe long (vago-vagal) duodéno-gastrique, c'està-dire par la suppression d'un contrôle inhibiteur de l'activité antrale comme il a été suggéré en première analyse (Darcy et al., 1979). Cette libération de l'activité antrale impliquerait en effet, dans la conception classique du rôle propulsif de la pompe antro-pylorique, une évacuation gastrique accrue en l'absence de toute inhibition rétrograde, ce que démentent les résultats. Par contre, leur interprétation peut être envisagée dans le cadre de la conception actuelle de la mécanique d'évacuation (Laplace, 1982) qui prend en compte de façon distincte le rôle de forces positives (pression intra-gastrique et/ou activité antrale selon les caractéristiques physiques des contenus) et celui des forces négatives ou résistances au débit de sortie de l'estomac (pylore) et au débit d'entrée dans l'intestin.

\section{Hypothèses explicatives du ralentissement de l'évacuation gastrique.}

La partie proximale de l'estomac est le siège du phénomène de relaxation réceptive qui repose sur un réflexe vago-vagal prenant origine au niveau du pharynx, de l'œesophage et de l'estomac, et dont l'arc efférent est constitué par des fibres inhibitrices non adrénergiques, non cholinergiques (Jahnberg et al., $1977 \mathrm{a}$ et b). Lors de dénervation de l'estomac proximal, il y a augmentation importante de la pression intracavitaire lorsque croît le volume introduit dans l'estomac (Jahnberg et al., 1975). Ce phénomène, vérifié chez l'Homme, le Chien et le Chat, est également vrai chez le Porc (Stadaas, Aune et Haffner, 1974). La pression intragastrique étant la principale force responsable de l'évacuation des liquides, celle-ci est accrue lorsque la relaxation réceptive est supprimée (Donovan et al., 1974 ; Berger et al., 1976 ; Mac Gregor, Parent et Meyer, 1977). La déafférentation totale réalisée chez nos porcs préserve la moitié seulement des afférences d'origine pharyngée et œsophagienne, et supprime les afférences d'origine gastrique. Dès lors, l'amoindrissement probable de la relaxation réceptive entraînerait, selon les caractéristiques physiques des contenus gastriques, soit une accélération de l'évacuation des contenus gastriques totaux (démentie par les résultats), soit une accélération de l'évacuation de la phase liquide. Cette seconde hypothèse est seule compatible avec la teneur en matière sèche nettement plus élevée chez les déafférentés et la plus grande ampleur de la rétention en termes de matière sèche que de matière fraîche.

En ce qui concerne le rôle de l'antre et de sa motricité, deux aspects doivent être pris en compte. D'une part, l'activité antrale augmente avec la taille du repas et décroît parallèlement au déroulement de l'évacuation gastrique chez le chien (Stemper et Cooke, 1975) comme chez le porc (Laplace, 1980b). D'autre part, l'activité antrale dépend très largement de la structure physique de l'aliment : l'évacuation est importante mais la motricité antrale faible pour un repas homogénéisé alors que le même régime sous forme solide-liquide conduit à une forte acti- 
vité antrale et à des réponses sécrétoires sensiblement différentes (Rees, Go et Malagelada, 1979 ; Malagelada, Go et Summerskill, 1979). La même variation d'intensité d'activité antrale existe chez le porc pour des régimes standard grossiers par rapport à des régimes semi-purifiés (Laplace, 1980b). La fonction de l'antre est en fait d'exprimer les suspensions de fines particules susceptibles d'évacuation à l'instar des liquides (Hinder et Kelly, 1977 ; Kelly, 1980) et de retenir et triturer les solides pour en assurer la liquéfaction (Meyer et al., 1979 ; Meyer, 1980). Ce rôle de filtre sélectif est vérifié chez le porc (Clémens et al., 1975) comme chez l'Homme (Malagelada, 1977 ; Holt et al., 1982). Mais le fait est que l'activité antrale, dans son intensité et sa durée apparaît plus comme déterminée par le degré de réplétion (bilan taille du repas - évacuation gastrique) et les caractéristiques physiques du contenu intragastrique, que comme déterminante de l'intensité de l'évacuation (Laplace, 1982). II est donc plausible que la prolongation de l'activité antrale postprandiale chez les sujets déafférentés ne soit que la conséquence de l'évacuation gastrique lente enregistrée chez ces animaux. La rétention peut être globalement exprimée en termes de matière sèche dans la mesure où les profils d'évacuation de l'amidon et de l'azote après déafférentation coïncident bien avec ceux d'évacuation de la matière sèche. Cette particularité justifie la mesure exclusive des quantités de matière sèche dans la seconde série expérimentale, et témoigne de l'absence d'une évacuation différentielle des constituants de l'aliment. Par contre, il y a bien évacuation différentielle des solides (matière sèche) par rapport aux liquides.

Dès lors que l'on reconnaît à l'antre un rôle essentiel de trituration, la rétention relative des solides pourrait résulter d'une certaine inefficacité de l'activité antrale. De fait, des désordres consécutifs aux vagotomies tronculaires ou de branche ont été décrits chez diverses espèces : perturbation de l'électrogenèse et de la propagation des activités de contrôle (Nelsen et al., 1967; Kelly et Code, 1969 ; Papasova et Atanassova, 1972 ; Miolan, 1974 ; Stoddard, Smallwood et Duthie, 1981), anomalies observées également chez le Porc après vagotomie tronculaire bilatérale ou déafférentation totale (Darcy, Laplace et Villiers, 1979) ; réduction de l'activité motrice antrale (Nagaoka, 1968 ; Kelly et Code, 1969 ; Wilbur et Kelly, 1973 ; Stoddard et al., 1973 ; Bone et al., 1977a) ; défaut de coordination des activités antrales et duodénales (Stoddard et Duthie, 1976). Toutes ces anomalies sont communément associées à un ralentissement de l'évacuation des solides (Kalbasi et al., 1975 ; Mroz et Kelly, 1977 ; Mac Gregor, Martin et Meyer, 1977). II s'avère donc possible qu'existent chez nos animaux déafférentés des désordres analogues de l'activité antrale, affectant la faculté de liquéfaction et donc d'évacuation d'un aliment solide. Dès lors, l'augmentation de la durée d'activité postprandiale ne serait que la conséquence de la rétention gastrique ainsi induite.

Enfin, troisième hypothèse, la rétention gastrique serait-elle également imputable à une modification des résistances à l'écoulement, de sortie de l'estomac (pylore) et d'entrée dans l'intestin ? La résistance au remplissage du duodénum est bien établie par divers travaux (Weisbrodt et al., 1969 ; Bone et al., 1977b ; Miller et al., 1981). Le rôle du pylore fait par contre l'objet d'une controverse alimentée par les études cliniques ou expérimentales, in vitro ou in vivo. La diversité 
des opinions sur ce point peut être rapprochée du fait que l'évacuation gastrique nette est, lors de myectomie pylorique, largement modifiée par une augmentation considérable des reflux duodéno-gastriques, ce qui peut multiplier par 7 le pourcentage de contenus évacués plus d'une fois (Müller-Lissner et al., 1982). Mais en définitive, l'existence d'une résistance pylorique au débit de sortie de l'estomac s'avère très réelle (Miller et al., 1981). Cette résistance augmente sous l'influence de facteurs classiquement inhibiteurs de l'évacuation (Fisher et Cohen, 1973) et l'injection d'huile d'olive dans le duodénum peut conduire à une quasi fermeture du pylore (Aste et Molinari, 1976). Une certaine augmentation de la résistance pylorique (et éventuellement duodénale) à l'évacuation des contenus gastriques pourrait donc expliquer la rétention observée après déafférentation. De fait, divers travaux chez le chien (Mir et al., 1979), le chat (Edin, 1980) et le lapin (Deloof et Rousseau, 1980 ; Deloof, 1981) suggèrent l'existence d'une innervation vagale inhibitrice du pylore, non cholinergique et non adrénergique, dont la suppression pourrait conduire à un pylorospasme. La présence dans le nerf grand splanchnique d'un contingent de fibres cholinergiques excitatrices pour la musculature du pylore (Lerman et al., 1981 ; Lidberg et al., 1982) ne pourrait que renforcer cet effet d'occlusion pylorique par déséquilibre vago-sympathique. Enfin, le fait que certains neurones moteurs vagaux distribuent leurs fibres efférentes simultanément à l'antre et au duodénum (Kraglund, Schroder et Jorgensen, 1983) suggère que la rétention pourrait aussi résulter d'une dyscoordination de la jonction gastroduodénale.

\section{Conclusions.}

La déafférentation vagale totale entraîne chez le Porc un ralentissement considérable de l'évacuation gastrique. Celui-ci résulte bien, pour l'essentiel, de la privation d'afférences, alors que l'inévitable réduction concomitante du volume d'efférences ne joue qu'un rôle limité. Le ganglion plexiforme gauche paraît jouer un rôle fonctionnel plus important que son homologue contralatéral à l'égard de l'effecteur gastro duodénal. La rétention gastrique s'exerce plus particulièrement à l'égard de la phase solide des contenus. Les modifications de l'évacuation de l'estomac relèvent probablement de plusieurs facteurs tels que moindre faculté de relaxation réceptive, perturbation de l'activité antrale et dans une certaine mesure pylorospasme. II paraît également vraisemblable que la déafférentation réduise le volume de la sécrétion gastrique postprandiale.

Reçu en mai 1983.

Accepté en juin 1984.

\section{Références}

ANDREWS P. L. R., GRUNDY D., SCRATCHERD T., 1980. Vagal afferent discharge from mechano receptors in different regions of the ferret stomach. J. Physiol. (London), 298, 513-524.

ASTE H., MOLINARI F., 1976, Gastric reflux measurements during duodenal infusion with saline, acid and fat. Digestion, 14, 184-187. 
BERGER T., CEDER L., HAMFELT A., MEURLING S., 1976. Effect of highly selective vagotomy on gastric emptying. Scand. J. Gastroent., 11, 829-832.

BONE J., BRANDSBORG O., BRANDSBORG M., MIKKELSEN K., AMDRUP E., 1977a. Antral denervation in parietal cell vagotomized dogs. Effect upon gastric emptying and motility, Heidenhain pouch acid secretion and serum gastrin concentration. Digestion, 15, 1-8.

BONE J., ORNSHOLT J., BRANDSBORG O., BRANDSBORG M., MIKKELSEN K., 1977b. Duodenal denervation in dogs. Effect upon gastroduodenal emptying and motility, Heidenhain pouch acid secretion and serum gastrin concentration. Digestion, 15, 129-135.

CLEMENS E. T., STEVENS C. E., SOUTHWORTH M., 1975. Sites of organic acid production and pattern of digesta movement in the gastro intestinal tract of swine. J. Nutr., 105, 759-768.

COOKE A. R., 1975. Control of gastric emptying and motility. Gastroenterology, 68, 804-816.

CUBER J. C., LAPLACE J. P., 1979. Evacuation gastrique de l'amidon et de l'azote d'un régime semi-purifié à base d'amidon de maïs chez le Porc. Ann. Zootech., 28, 173-184.

CUBER J. C., LAPLACE J. P., VILLIERS P. A., 1980. Fistulation de l'estomac et contenus gastriques résiduels après ingestion d'un régime semi-purifié à base d'amidon de maïs chez le Porc. Reprod. Nutr. Dévelop., 20, 1161-1172.

DARCY B., FALEMPIN M., LAPLACE J. P., ROUSSEAU J. P., 1979. Importance de la voie vagale sensitive : recherche d'une technique de déafférentation sélective chez le Porc et le Mouton. Ann. Biol. anim. Bioch. Biophys., 19, 881-888.

DARCY B., LAPLACE J. P., VILLIERS P. A., 1979. Note : Altérations du rythme électrique de base de l'antre gastrique après diverses vagotomies chez le Porc. Ann. Zootech., 28, 465-471.

DELOOF S., 1981. Effets de la vagotomie thoracique sur l'activité électromyographique antropylorique chez le lapin. Reprod. Nutr. Dévelop., 21, 776.

DELOOF S., ROUSSEAU J. P., 1980. Influence du nerf vague sur l'activité électromyographique de la région antropylorique chez le lapin. Reprod. Nutr. Dévelop., 20, 1149-1154.

DONOVAN I. A., GUNN I. F., BROWN A., ALEXANDER-WILLIAMS J., CLARKE R. J., 1974. A comparison of gastric emptying before and after vagotomy with antrectomy and vagotomy with pyloroplasty. Surgery, 76, 729-732.

EDIN R., 1980. The vagal control of the pyloric motor function. A physiological and immunohistochemical study in cat and man. Acta physiol. scand., Suppl. 485, $30 \mathrm{pp}$.

EL OUAZZANI T., MEI N., 1979. Mise en évidence électrophysiologique des thermo-récepteurs vagaux dans la région gastro-intestinale. Leur rôle dans la régulation de la motricité digestive. Exp. Brain Res., 34, 419-434.

EL OUAZZANI T., MEI N., 1981. Acido et glucorécepteurs vagaux de la région gastroduodénale. Exp. Brain Res., 42, 442-452.

FALEMPIN M., ROUSSEAU J. P., 1979. Vagal digestive deafferentation in sheep. Ann. Rech. vét., 10, 186-188.

FISHER R. S., COHEN S., 1973. Physiological characteristics of the human pyloric sphincter. Gastroenterology, 64, 67-75.

HINDER R. A., KELLY K. A., 1977. Canine gastric emptying of solids and liquids. Am. J. Physiol., 233, E335-E340.

HOLT S., REID J., TAYLOR T. V., TOTHILL P., HEADING R. C., 1982. Gastric emptying of solids in man. Gut, 23, 292-296.

IGGO A., 1955. Tension receptors in the stomach and the urinary bladder. J. Physiol. (London), 128. 593-607.

IGGO A., 1957. Gastric mucosal chemoreceptors with vagal afferent fibres in the cat. Quart. J. exp. Physiol., 42, 398-409.

JAHNBERG T., ABRAHAMSSON H., JANSSON G., MARTINSON J., 1977a. Vagal gastric relaxation in the dog. Scand. J. Gastroent, 12, 221-224.

JAHNBERG T., ABRAHAMSSON H., JANSSON G., MARTINSON J., 1977b. Gastric relaxatory response to feeding before and after vagotomy. Scand. J. Gastroent., 12, 225-228.

JAHNBERG T., MARTINSON J., HULTEN L., FASTH S., 1975. Dynamic gastric response to expansion before and after vagotomy. Scand. J. Gastroent., 10, 593-598.

JEANNINGROS R., MEI N., 1980. Données préliminaires sur la réponse des chémorécepteurs intestinaux aux acides aminés. Reprod. Nutr. Dévelop., 20, 1615-1619.

KALBASI H., HUDSON F. R., HERRING A., MOSS S., GLASS H. I., SPENCER J., 1975. 
Gastric emptying following vagotomy and antrectomy and proximal gastric vagotomy. Gut, 16, 509-513.

KELLY K. A., 1980. Gastric emptying of liquids and solids : role of proximal and distal stomach. Am. J. Physiol., 239, G71-G76.

KELLY K. A., CODE C. F., 1969. Effect of transthoracic vagotomy on canine gastric electrical activity. Gastroenterology, 57, 51-58.

KRAGLUND K., SCHRODER H. D., JORGENSEN S., 1983. Neural connections between antrum and duodenum. Experientia, 39, 869-870.

LAPLACE J. P., 1980a. Surgical deprivation of vagal afferences from the gastrointestinal tract of the pig: Clinical and EMG studies, 145-152. In CHRISTENSEN J., Gastrointestinal motility, Raven Press, New York.

LAPLACE J. P., 1980b. Stomach and small intestine motility in the pig : electromyography in nutritional studies, 24-47, In LOW A. G., PARTRIDGE I. G., Current concepts of digestion and absorption in pigs, NIRD Press, Reading.

LAPLACE J. P., 1982. Fonction de l'estomac chez le porc : révision des concepts usuels sur son rôle dans la digestion et la mécanique de son évacuation, 29-44. In LAPLACE J. P., CORRING T., RÉRAT A., Physiologie digestive chez le porc, INRA-Pub. Ed. (Les Colloques de I'INRA, $n^{\circ} 12$ )

LAPLACE J. P., CUBER J. C., VILLIERS P. A., 1979. Note : Evacuation gastrique après déafférentation vagale subtotale chez le Porc. Ann. Zootech., 28, 459-463.

LEEK B. F., 1977. Abdominal and pelvic visceral receptors. Br. med. Bull., 33, 163-168.

LERMAN S. H., MASON G. R., BATHON E. M., ORMSBEE H. S., 1981. Pyloric motor response to sympathetic nerve stimulation in dogs. Surgery, 89, 460-465.

LIDBERG P., EDIN R., LUNDBERG J. M., DAHLSTRÖM A., KEWENTER J., AHLMAN H., 1982. Evidence for a non classical splanchnic neural control of the feline pylorus. Acta physiol. scand., 115, 225-232.

MAC GREGOR I. L., MARTIN P., MEYER J. H., 1977. Gastric emptying of solid food in normal man and after subtotal gastrectomy and truncal vagotomy with pyloroplasty. Gastroenterology, 72, 206-211.

MAC GREGOR I., PARENT J., MEYER J. H., 1977. Gastric emptying of liquid meals and pancreatic and biliary secretion after subtotal gastrectomy or truncal vagotomy and pyloroplasty in man. Gastroenterology, 72, 195-205.

MALAGELADA J. R., 1977. Quantification of gastric solid-liquid discrimination during digestion of ordinary meals. Gastroenterology, 72, 1264-1267.

MALAGELADA J. R., GO V. L. W., SUMMERSKILL W. H. J., 1979. Different gastric, pancreatic, and biliary responses to solid-liquid or homogenized meals. Dig. Dis. Sci., 24, 101-110.

MEI N., 1970a. Disposition anatomique et propriétés électrophysiologiques des neurones sensitifs vagaux chez le chat. Exp. Brain Res., 11, 465-479.

MEI N., 1970b. Mécanorécepteurs vagaux digestifs chez le chat. Exp. Brain Res., 11, 502-514.

MEI N., 1978. Vagal glucoreceptors in the small intestine of the cat. J. Physiol. (London), 282, 485-506.

MEI N., 1981. La sensibilité viscérale. J. Physiol. (Paris), 77, 597-612.

MEI N., CONDAMIN M., BOYER A., 1980. The composition of the vagus nerve of the cat. Cell Tissue Res., 209, 423-431.

MEYER J. H., 1980. Gastric emptying of ordinary food : effect of antrum on particle size. Am. J. Physiol., 239, G133-G135.

MEYER J. H., THOMSON J. B., COHEN M. B., SHADCHEHR A., MANDIVLA S. A., 1979. Sieving of solid food by the canine stomach and sieving after gastric surgery. Gastroentero$\log y, 76,804-813$.

MILLER J., KAUfFMAN G., ELASHOFF J., OHASHI H., CARTER D., MEYER J. H., 1981. Search for resistances controlling canine gastric emptying of liquid meals. Am. J. Physiol., 241, G403-G415.

MIOLAN J. P., 1974. La motricité de l'estomac et du sphincter œsophagien inférieur, étude électromyographique; rôle de l'innervation extrinsèque. Thèse Doct. Spécial, Physiol. Anim., Univ. Aix-Marseille III. 
MIR S. S., TELFORD G. L., MASON G. R., ORMSBEE H. S., 1979. Non cholinergic non adrenergic inhibitory innervation of the canine pylorus. Gastroenterology, 76, 1443-1448.

MROZ C. T., KELLY K. A., 1977. The role of the extrinsic antral nerves in the regulation of gastric emptying. Surg. Gynecol. Obstet., 145, 369-377.

MÜLLER-LISSNER S. A., SONNENBERG A., SCHATTENMANN G., HOLLINGER A., SIEWERT J. R., BLUM A. L., 1982. Gastric emptying and postprandial duodenogastric reflux in pylorectomized dogs. Am. J. Physiol., 242, G9-G14.

NAGAOKA K., 1968. Electromyographic study on the mechanism of delayed gastric emptying after vagotomy in dogs. Tohoku J. exp. Med., 95, 1-13.

NELSEN T. S., EIGENBRODT E. H., KEOSHIAN L. A., BUNKER C., JOHNSON L., 1967. Alterations in muscular and electrical activity of the stomach following vagotomy. Arch. Surg., 94, 821-835.

PAPASOVA M., ATANASSOVA E., 1972. Changes in the bioelectric activity of the stomach after bilateral transthoracal vagotomy. Bulgarian Acad. Sci., 14, 121-133.

REES W. D. W., GO V. L. W., MALAGELADA J. R., 1979. Antroduodenal motor response to solid-liquid and homogenized meals. Gastroenterology, 76, 1438-1442.

ROZÉ C., 1980a. Régulation de la motricité œesophagienne et gastrique. Aspects physiologiques et pharmacologiques récents. 2) Estomac. Gastroenterol. clin. biol., 4, 600-615.

ROZÉ C., 1980b. Neurohumoral control of gastrointestinal motility. Reprod. Nutr. Dévelop., 20, $1125-1141$.

ROZÉ C., COUTURIER D., CHARIOT J., DEBRAY C., 1977. Inhibition of gastric electrical and mechanical activity by intraduodenal agents in pigs and the effects of vagotomy. Digestion, 15, 526-539.

STADAAS J., AUNE S., HAFFNER J. F. W., 1974. Effects of proximal gastric vagotomy on intragastric pressure and adaptation in pigs. Scand. J. Gastroent., 9, 479-485.

STEMPER T. J., COOKE A. R., 1975. Gastric emptying and its relationship to antral contractile activity. Gastroenterology, 69, 649-653.

STODDARD C. J., DUTHIE H. L., 1976. Effect of vagotomy on the response of gastric myoelectrical activity to glucagon and food. Scand. J. Gastroent., 11, (suppl. 42), 77-84.

STODDARD C. J., SMALLWOOD R. H., DUTHIÉ H. L., 1981. Electrical arrhythmias in the human stomach. Gut, 22, 705-712.

STODDARD C. J., WATERFALL W. E., BROWN B. H., DUTHIÉ H. L., 1973. The effects of varying the extent of the vagotomy on the myoelectrical and motor activity of the stomach. Gut, 14, 657-664.

WEISBRODT N. W., WILEY J. N., OVERHOLT B. F., BASS P., 1969. Relation between gastroduodenal muscle contractions and gastric emptying. Gut, 10, 543-548.

WILBUR B. G., KELLY K. A., 1973. Effect of proximal gastric, complete gastric, and truncal vagotomy on canine gastric electric activity, motility and emptying. Ann. Surg., 178, 295303. 